405 Coffey Hall, 1420 Eckles Ave, St. Paul, MN, 55108-6068; telephone (800) 367-5363 or fax (612) 625-2207; E-mail tbenson@mes.umn.edu.

\section{Infections Linked to Anesthetic}

The CDC recently reported the results of investigations conducted between June 1990 and February 1993 at seven hospitals following unusual outbreaks of bloodstream infections, surgical wound infections, and acute febrile episodes after surgical procedures. A case-control study identified 62 patients that had an organism-specific infection or acute febrile episode after a surgical procedure during the study period.

Only exposure to propofol, a lipid-based anesthetic agent, was associated significantly with the postoperative complications. In six of the outbreaks, an etiologic agent (Staphylococcus aureus, Candida albicans, Moraxella osloensis, Enterobacter agglomerans, or Serratia marcescens) was identified, and the same strains were isolated from the case patients.

Although cultures of unopened containers of propofol were negative, cultures at two hospitals of propofol from syringes currently in use were positive. At one hospital, the recovered organism was identical to the organism isolated from the case patients. Interview and observation of anesthesiology personnel documented lapses of aseptic technique and multiple opportunities for extrinsic contamination. These included (1) preparation of multiple syringes of propofol at one time for use throughout the day; (2) reuse of syringes or infusion-pump lines, or both, on different patients; (3) use of syringes of propofol that had been prepared up to 24 hours beforehand; (4) transfer of prepared syringes of propofol between operating rooms or facilities; (5) failure to wear gloves during procedures involving touching of the mucous membrane or preparing or administering propofol; and (6) failure to disinfect the rubber stoppers of 50-mL propofol vials before use.

The authors note that despite written recommendations of professional associations such as the American Society of Anesthesiology that specifically advocate the use of aseptic techniques during handling of medications, several reports have been published on poor compliance with aseptic techniques and infection control practices by anesthesia personnel.

Despite earlier reports of propofol-associated outbreaks in the 1990s and the education efforts by the manufacturer, the number of clusters of infections or fever associated with propofol use reported to the FDA rose steadily from 1991 through 1993. The authors suggest that further efforts are needed to reduce the risk associated with propofol use, including increased efforts to educate anesthesia personnel and to restrict propofol access to those educated in its unique properties and handling requirements. The authors note that with the increasing use of lipid-based medications, which support rapid bacterial growth at room temperature, strict aseptic techniques are essential during the handling of these agents to prevent extrinsic contamination and dangerous infectious complications.

FROM: Bennett SN, McNeil MM, Bland LA, et al. Postoperative infections traced to contamination of an intravenous anesthetic, propofol. $N$ Engl $J$ Med 1995;333:147-54.

\section{Hepatitis A From Patient to Staff}

An outbreak of hepatitis A recently was reported in a Florida children's hospital. The case patient, a 14-monthold girl with severe B-cell immunodeficiency, hypogammaglobulinemia, and undiagnosed hepatitis A (anti-HAV negative), transmitted hepatitis A to 26 hospital staff, inpatients, and household contacts via gross fecal contamination from prolonged diarrhea. Infection was proven in the index case by PCR of stool and serum. All infected employees had direct contact with the infected patient, and the greatest risk of infection was for those who handled the source patient's bedpan, diaper, or gown. Glove use was not associated with a decreased risk of infection.

Of the 250 employees tested for hepatitis A virus infection, $60 \%$ were susceptible and had exposure to the suspected source patient. Attack rates ranged from 7\% for physicians to $18 \%$ for nurses. The overall attack rate was $13 \%$ for all hospital staff.

FROM: Burkholder BT, Coronado VG, Brown J, et al. Nosocomial transmission of hepatitis A in a pediatric hospital traced to an anti-hepatitis A virus-negative patient with immunodeficiency. Pediatr Infect Dis J 1995;14: 261-266.

Additional news items in this issue: NIOSH to Issue User Guidelines, page 498; OSHA Updates Free Catalog of Safety and Training Materials, page 502; Increase in Vacomycin-Resistant Enterococci, page 505; Senate Votes on Ryan White Act, page 511; Safety Devices Reduce Injuries, page 517; Rules on HIV Testing of Source Patients, page 525; Hepatitis B Linked to Cryopreservation Tank, page 531 